\title{
The principle of gravity-inertial orientation
}

\author{
Victor Dmitriev ${ }^{1}$, and Ruslan Frolov ${ }^{1, *}$ \\ ${ }^{1}$ National Research Tomsk Polytechnic University, 634050 Tomsk, Russia
}

\begin{abstract}
The important problem of gyrocompass without drift creation is considered in this article. The designing device allows to define the accurate information about mobile and stationary objects orientation in the case of long-term (months, years) absence of the determining the cardinal direction possibility. This article focuses on the idea of gyrocompass without drift creation, which works on the principle of gravity-inertial orientation at the theoretical level.
\end{abstract}

\section{Introduction}

Ground orientation is the position indication of man and other different objects on the ground relative to the cardinal directions and landmarks. The science of navigation considers these issues. In modern science and technology, interdisciplinary ideas have the greatest development.

\section{Formulation of the problem}

Nowadays, the theory of inertial navigation systems emerged as the independent branch of applied mechanics with its objectives and methods. To have a gyrocompass with minimal drift, and ideally - without drift is the principal technical problem, which has always been and is currently exist in the field of navigation systems [1-4].

\section{Technical research}

Gyroscopic action of the rapidly spinning bodies consists in their capacity to resist to the external forces, changing their position in the inertial space [5-7].

The apparent anomaly in motion of the gyroscope is explained by the appearance of Coriolis (complementary) acceleration.

Coriolis acceleration occurs when the relative and translational motion are simultaneously applied to the object (point), and consequently Coriolis force acts on the object, located on the Earth surface:

$$
F_{c o r}=m \cdot \dot{v}_{C}=2 \cdot m \cdot \ddot{v}_{C} \cdot \Omega_{E} \cdot \cos \varphi
$$

\footnotetext{
*Corresponding author: phrolov1994@mail.ru
} 
where, $m$ - mass of the object; $\Omega_{\mathrm{E}}-$ the angle rate of Earth's rotation; $\dot{v}_{C}-$ Coriolis (complementary) acceleration.

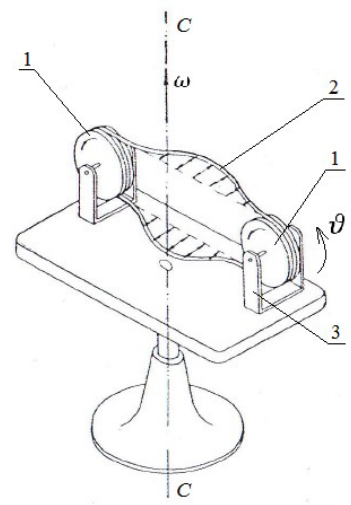

Fig. 1. Installation for Coriolis acceleration demonstration.

Figure 1 shows the main parts of installation, they are 1 - pulley, 2 - belt, 3 - table. Pulleys 1 start to rotate with fixed table. In this case, the straight sections of the belt will move along table with some relative rate. If the table is forced to rotate around the $\mathrm{C}-\mathrm{C}$ axis with the rate $\omega$, interaction of the inertial and elastic forces of the belt will provide the parabolic character of the belt deformation. To be more convincing, that it is not the action of the centrifugal forces, but it is the action of the Coriolis acceleration, $\mathrm{C}-\mathrm{C}$ axis does not coincide with the axis of the table rotation [8].

A similar pattern is observed, if the undulatory motion of the object with weight $m$ along the place vertical is made. In this case, the object acquires the linear velocity $\vartheta_{r}$ along $\bar{g}$. The value of linear velocity in time is represented by the expression:

$$
\mathrm{v}_{r}=\omega \cdot z_{m} \cdot \cos \omega t
$$

where, $\mathrm{z}_{\mathrm{m}}$ - amplitude of swell; $\omega$ - pulsatance; $\mathrm{t}$ - time.

Then (Figure 2), there are two motions: the rotational (translational) from the Earth's rotation and the linear (relative) which acts along the place vertical. Consequently, the compass force $F_{c o m}$ appears [9], which can be written as:

$$
F_{c o m}=2 \cdot m \cdot \Omega_{E} \cdot \cos \phi \cdot \omega \cdot z_{m} \cdot \cos \omega t,
$$

where, $m$ - mass of the sensitive element; $\Omega_{\mathrm{E}}$ - the angle rate of Earth's rotation; $\varphi$ - site latitude; $z_{m}$ - amplitude of swell; $\omega$ - pulsatance of the periodic motion of the sensitive element.

Consider the general case of the point (sensitive element) rectilinear motion in the mechanical system, which has three degrees of freedom (Figure 2).

Applying the Lagrange method, we will have the differential equations, which describe forced movement of the sensitive element in vertical direction. Kinetic energy $T$, potential energy $V$, scattering function $F$, generalized forces $\left(Q_{P}, Q_{R}, Q_{F}\right)$ are determined for this mechanical system as: 


$$
\begin{array}{cc}
T=\frac{1}{2} m \cdot \dot{q}^{2}, & Q_{P}=-\frac{\partial P}{\partial q}=-c q, \\
V=\frac{1}{2} c \cdot q, & Q_{R}=-\frac{\partial F}{\partial \dot{q}}=-k_{D} \cdot \dot{q}, \\
F=\frac{1}{2} k_{D} \dot{q}^{2}, & Q_{F}=\frac{d}{d t}\left(\frac{\partial T}{\partial \dot{q}}\right)=2 \cdot m \cdot \Omega_{E} \cdot \cos \varphi \cdot \omega \cdot z_{m} \cdot \cos \omega t .
\end{array}
$$

Imagine that point (object) is placed in the force field above the Earth's surface and this point is forced to move vertically along $\mathrm{Y}$ axis. In accordance with the above information, the periodically compass force $F_{c o m}$ will act on this point in the East-West direction. Let us consider the dynamics of the sensitive element motion.

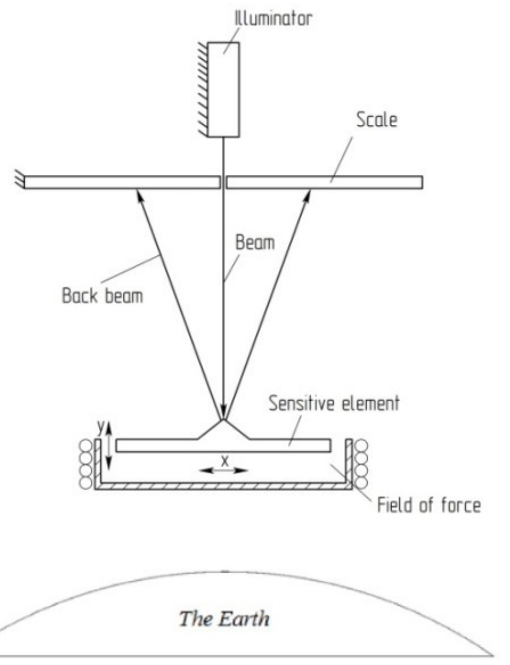

Fig. 2. Dynamics of the sensitive element motion.

We will obtain the expression (5) if the values of all variables are substituted in the Lagrange equations:

$$
m \ddot{q}=-c q-k_{D} \dot{q}+H \cdot \cos \omega t
$$

where $H=2 \cdot m \cdot \Omega_{E} \cdot \cos \varphi \cdot \omega \cdot z_{m}$

If we divide both sides of (5) by the mass, it will have a conventional view:

$$
\ddot{q}+2 n \dot{q}+k^{2} q=h \cdot \cos \omega t
$$

where $\frac{c}{m}=k^{2}, \quad \frac{k_{D}}{m}=2 n, \quad \frac{H}{m}=h$

The solution of this equation is the sum of homogeneous $\mathrm{q}_{1}$ and particular solutions $q_{2}$.

The solution of the homogeneous equation $q_{1}$ is given by:

$$
q_{1}=A_{1} e^{-n t} \sin k_{1} t
$$

where $k_{1}=\sqrt{k^{2}-n^{2}}$. 
Equation (7) determines the self-oscillation of the point (object).

In the amplitude form the equation (8) has the form:

$$
q_{2}=A_{2} \sin (\omega t-\varepsilon)
$$

where $A_{2}$ - amplitude of forced oscillations; $\varepsilon$ - phase angle of forced oscillations relative phase of the exciting force

Let us consider the forced oscillations (8), defined by the equation (8). It is necessary to replace in the expression (6) $q$ to $q_{2}$, and to determine the first and second derivative. And if we do a number of required mathematical operations, a particular solution of the forced oscillations equation can be written as:

$$
q_{2}=\frac{h}{\sqrt{\left(k^{2}-\omega^{2}\right)+4 n^{2} \omega^{2}}} \sin (\omega t-\varepsilon)
$$

To solve the proposed problem we consider the forced oscillations (9). The amplitude of these oscillations always direct to the East-West direction and is determined by the forced oscillations in the direction of the place vertical with the value of the amplitude $H$ and the frequency $\omega$. The frequency $\omega$ is necessary to select by varying the damping factor $k_{D}$, thus amplitude $H$. will be adjusted In accordance with the above information, the periodically complementary acceleration exists. Consequently the periodically motion with permanent direction East-West appears.

\section{Conclusion}

In this paper, theoretical substantiation and schematic solution of the practical implementation of the gravity-inertial gyrocompass without drift are presented. This gyrocompass can permanently monitor the East-West direction of the object regardless of time and it basing site.

\section{References}

1. G. Shvecov, et al Gravitacionno-inercialnoe orientirovanie (publishing TPU, Tomsk, Russia, 2004) (in Russian)

2. A.V. Taracenko, V.S. Dmitriev, T.G. Kostuchenko, KORUS, 83 (2004)

3. V. Dmitriev, Y. Britova, MATEC Web of Conferences 48, 01005 (2016)

4. Yu. Britova, V. Dmitriev, T. Kostyuchenko, IOP Conference Series: Materials Science and Engineering 132(1), 012011 (2016)

5. V.S. Volkova, V.I. Kopytov, 5th Korea-Russia International Symposium on Science and Technology, 29 (2001)

6. V.S. Ivanova, 8th International Scientific and Practical Conference of Students, Postgraduates and Young Scientists; "Modem Techniques and Technologies", 42 (2002)

7. P. Baranov, T. Nesterenko, E. Tsimbalist, E. Barbin, A. Koleda, S. Vtorushin, IMEKO: Proc. 14th IMEKO TC10 Workshop on Tech.l Diag. (2016)

8. V. Pavlov, Teoriya giroskopa i giroskopicheskih priborov (Sudostroenie, Leningrad, Russia, 1964) (in Russian)

9. G. Shvecov, USSR Patent № 4672218 (1991) (in Russian) 